\title{
Solar flare mechanism based on magnetic arcade reconnection and island merging
}

\author{
C. Z. Cheng and G. S. Choe \\ Princeton Plasma Physics Laboratory, Princeton University, Princeton, NJ 08543-0451, U.S.A.
}

(Received May 29, 2000; Revised November 28, 2000; Accepted November 28, 2000)

\begin{abstract}
We propose a model describing physical processes of solar flares based on resistive reconnection of magnetic field subject to continuous increase of magnetic shear in the arcade. The individual flaring process consists of magnetic reconnection of arcade field lines, generation of magnetic islands in the magnetic arcade, and coalescence of magnetic islands. When a magnetic arcade is sheared (either by footpoint motion or by flux emergence), a current sheet is formed and magnetic reconnection can take place to form a magnetic island. A continuing increase of magnetic shear can trigger a new reconnection process and create a new island in the underlying arcade below the magnetic island. The newborn island rises faster than the preceding island and merges with it to form one island. Before completing the island merging process, the newborn island exhibits two phases of rising motion: a first phase with a slower rising speed and a second phase with a faster rising speed. The flare plasma heating occurs mainly due to magnetic reconnection in the current sheet under the newborn island. The newborn island represents the X-ray plasma ejecta which shows two phases of rising motion observed by Yohkoh (Ohyama and Shibata, 1997). The first phase with slower newborn island rising speed corresponds to the early phase of reconnection of line-tied field in the underlying current sheet and is considered as the preflare phase. In the second phase, the island coalescence takes place, and the underlying current sheet is elongated so that the line-tied arcade field reconnection rate is enhanced. This phase is interpreted as the impulsive phase or the flash phase of flares. The obtained reconnection electric field is large enough to accelerate electrons to an energy level higher than $10 \mathrm{keV}$, which is necessary for observed hard X-ray emissions. After merging of the islands is completed, magnetic reconnection continues in the current sheet under the integrated island for a longer period, which is considered as the main phase of flares. The sequence of all these processes is repeated with some time interval while a shear-increasing motion continues. We propose that these repetitive flaring processes constitute a set of homologous flares.
\end{abstract}

\section{Introduction}

Solar flares are intense, abrupt release of energy occurring usually in the vicinity of an active region where the magnetic field is stressed. A large flare can release more than $10^{32} \mathrm{erg}$ of energy in about an hour, which is regarded as a part of magnetic energy release. Based on the temporal evolution of the flare emission, a flare process can be divided into several phases (e.g., Kane, 1974; Priest, 1982). In the preflare phase, which lasts about 10 minutes before the flare onset, enhanced thermal emissions from the coronal plasma are detected mostly in the soft X-ray (photon energy $<10 \mathrm{keV}$ or $0.1 \mathrm{~nm}<\lambda<2 \mathrm{~nm}$ ). During the flash phase, which lasts typically about 5 minutes, the intensity and area of the emission rapidly increase. Then, in the main phase, the intensity slowly declines over about an hour and sometimes as long as a day. Large flares also exhibit an impulsive phase before the main phase, lasting 10-100 seconds, during which hard $\mathrm{X}$-ray $(\lambda<0.1 \mathrm{~nm})$ and microwave bursts are observed.

Flares were considered to occur due to either reconnection of open magnetic field lines above a magnetic arcade (Sturrock, 1968) or reconnection of stretched arcade field lines (Kopp and Pneuman, 1976). From Yohkoh observation Masuda et al. (1994) found that the HXR (hard X-ray)

Copy right (c) The Society of Geomagnetism and Earth, Planetary and Space Sciences (SGEPSS); The Seismological Society of Japan; The Volcanological Society of Japan; The Geodetic Society of Japan; The Japanese Society for Planetary Sciences. source, which is regarded to be directly related with magnetic reconnection, is located above the SXR (soft X-ray) loop. Later, Shibata et al. (1995) found that most flares observed by Yohkoh were associated with X-ray plasma ejecta (or plasmoid), some of which were launched well before the flare impulsive phase, and they inferred that the plasmoid ejection is not a consequence of the flare, but a cause of it. Based on these observations and the Kopp-Pneuman model (Kopp and Pneuman, 1976), Shibata (1998) proposed a plasmoid-induced reconnection model, in which a fast reconnection responsible for a flare is triggered by the plasmoid ejection. However, the formation and acceleration mechanism of the plasmoid was unaddressed in their model.

In the Kopp-Pneuman model the plasmoid, a magnetic island with helical field lines in 2D or a helical magnetic structure loosely connected to the solar surface in $3 \mathrm{D}$, is formed by reconnection of line-tied field lines in a magnetic arcade. This possibility has indeed been confirmed by several numerical simulations (Mikić et al., 1988; Inhester et al., 1992; Mikić and Linker, 1994; Linker and Mikić, 1995; Choe and Lee, 1996; Amari et al., 1996). In this picture, the rise of the plasmoid can be considered as a process of approaching a new equilibrium after a change in field topology. Thus, the plasmoid rising motion must eventually decelerate unless the reconnection of arcade field lines under the plasmoid continues indefinitely. However, recent 
observations of plasmoid ejection do not support the idea that the plasmoid is entirely driven by magnetic reconnection underneath the plasmoid. Ohyama and Shibata (1997) found from Yohkoh observations that an ejecta in an X-ray flare rises with a speed of $\sim 10 \mathrm{~km} / \mathrm{s}$ in the preflare phase, is accelerated to $\sim 130 \mathrm{~km} / \mathrm{s}$ for about 2 minutes just before the main peak of the HXR emission and then is accelerated during the impulsive phase to $\sim 200 \mathrm{~km} / \mathrm{s}$ for more than $7 \mathrm{~min}$. Excluding the short transition in the rising velocity, the X-ray ejecta motion shows two phases of rising velocity. Such two phases of rising velocity cannot be interpreted within the scope of the conventional Kopp-Pneuman picture although the flare morphology based on observations supports the Kopp-Pneuman-like field configuration in the vicinity of and under the reconnection site. A possible way of resolving this seeming contradiction is proposed in this paper.

In this paper, we present a flare model that can explain key observations of flaring processes including homologous flares. Our model is based on resistive magnetohydrodynamic (MHD) simulations of the evolution of a bipolar arcade due to magnetic reconnection and magnetic island creation by imposing various shear-increasing footpoint motions. In our study, we assume a closed initial arcade field configuration and focus on the evolution of the magnetic island not totally expelled from the sun. Because prominences are believed to reside at the bottom of a magnetic island (Kuperus and Raadu, 1974; Anzer, 1979), a newborn island can be considered as a prominence newly appearing in the solar atmosphere. When a magnetic island is formed by reconnection of an arcade field, the toroidal flux originally contained in line-tied flux tubes is redistributed into two flux systems: the magnetic island and the underlying arcade with the reconnected line-tied field. The magnetic shear is thus reduced in the underlying arcade after island formation. A further shearing motion increases the magnetic shear in the lower flux system, and above a critical value of shear magnetic reconnection takes place to form a new magnetic island. The newborn magnetic island then rises and merges with the overlying magnetic island to form a single integrated island. During this process, the newborn magnetic island exhibits two phases of rising motion with different speeds (a slower initial rising phase followed by a faster rising phase) corresponding to the observed two phases of the plasmoid (X-ray ejecta) motion reported by Ohyama and Shibata (1997). The reconnection electric field in the current sheet under the magnetic island system increases with the rising of the newborn island, reaches a maximum on the completion of island merging and gradually decreases for a longer duration. The obtained maximum reconnection electric field is large enough to accelerate electrons to an energy level higher than $10 \mathrm{keV}$, which is necessary for observed HXR emissions. Thus, the phase of the newborn island creation and its slow rising is regarded as the preflare phase, the fast newborn island rising phase involving the island coalescence is interpreted as the impulsive (or flash) phase, and the phase with a longer period of reconnection under the integrated island is considered as the main phase of a flare. The sequence of the above reconnection processes can be repeated as long as the magnetic shear is replenished, and we propose that a series of the reconnection sequence is a set of homologous flares.

\section{Simulation Model}

We investigate the plasma and magnetic field evolution in 2-1/2D bipolar magnetic arcades employing a $(x, y, z)$ Cartesian coordinate system. The magnetic arcade occupies the half-space $\{y>0\}$ and the plasma and magnetic field quantities are assumed to be invariant in the $z$-direction. The photosphere is modeled by the $y=0$ plane ignoring the curvature of the photosphere, and the polarity inversion line lies along the $z$-axis $(x=0, y=0)$. The magnetic field is assumed to be a potential field at $t=0$ and then evolves in response to the plasma flows in the solar surface. For simplicity, but without significant loss of generality, we assume that the initial field is symmetric across the $x=0$ plane and that the boundary flows on the solar surface are antisymmetric across the $z$-axis. Then, all the physical variables appearing in MHD description of plasma preserve a symmetry or an antisymmetry across the $x=0$ plane as well as an invariance in the $z$-coordinate for all the time.

The evolution of our model corona is governed by a full set of 2-1/2D MHD equations including gravity and resistivity. To fully treat the energetics of the solar corona, we need to consider the coronal heating, radiative cooling and anisotropic heat conduction (e.g., Choe and Lee, 1992). However, without a well established knowledge of the coronal heating mechanism and with the consideration of high thermal conductivity in the corona, the plasma is assumed to be isothermal. The gravity $\mathbf{g}=-g \hat{\mathbf{y}}$ is a function of $y$ given by $g(y)=G M_{\odot} /\left(R_{\odot}+y\right)^{2}=g_{0} R_{\odot}{ }^{2} /\left(R_{\odot}+y\right)^{2}$, where $G$ is the gravitational constant, $M_{\odot}$ the solar mass, $R_{\odot}$ the solar radius and $g_{0}=2.74 \times 10^{4} \mathrm{~cm} \mathrm{~s}^{-2}$ is the surface gravity. In our simulation, a constant kinematic viscosity $v=\mu / \rho=10^{-3}$ is used for the purpose of numerical smoothing.

We will consider a plasma with temperature of $2 \times 10^{6} \mathrm{~K}$. The magnetic field $\mathbf{B}$ is normalized by $B_{0}$ which is the maximum magnitude of the boundary normal field, the mass density $\rho$ by the initial density $\rho_{0}$ at the bottom boundary, the velocity $\mathbf{v}$ by $v_{0}=B_{0} /\left(4 \pi \rho_{0}\right)^{1 / 2}$, the time $t$ by $t_{0}=L_{0} / v_{0}$, and the resistivity $\eta$ by $L_{0} v_{0}$, where $L_{0}$ is the length unit. For $B_{0}=50 \mathrm{G}, \rho_{0}=1.9 \times 10^{-15} \mathrm{~g} / \mathrm{cm}^{3}$ (which is equivalent to a normalized electron density $\left.n_{0}=1.0 \times 10^{9} \mathrm{~cm}^{-3}\right)$ and $L_{0}=3.0 \times 10^{4} \mathrm{~km}$, we have $v_{0}=3.2 \times 10^{3} \mathrm{~km} / \mathrm{s}$ and $t_{0}=9.3 \mathrm{~s}$. The current density $\mathbf{J}$ is normalized by $J_{0}=$ $(c / 4 \pi)\left(B_{0} / L_{0}\right)=1.3 \times 10^{-4} \mathrm{~A} / \mathrm{m}^{2}$, and the normalized unit for electric field is $E_{0}=\left(v_{0} / c\right) B_{0}=1.6 \times 10^{4} \mathrm{~V} / \mathrm{m}$.

The invariance in $z$ in the $2-1 / 2 \mathrm{D}$ system allows the magnetic field to be expressed with only two scalar variables as $\mathbf{B}=\hat{\mathbf{z}} \times \nabla \psi+B_{z} \hat{\mathbf{z}}$, where the poloidal flux function $\psi$ is related to the the $z$-component of the vector potential by $\psi=$ $-A_{z}$. Because $\mathbf{B} \cdot \nabla \psi=0$, a magnetic field line lies in a constant $\psi$ surface. In equilibrium, one can show that $\mathbf{B} \cdot \nabla B_{z}=$ 0 , which means that the toroidal field $B_{z}$ is constant along a field line (see e.g., Cheng and Choe, 1998). This is a good approximation in a quasi-static evolution. Initially the magnetic field is in a hydrostatic equilibrium with the boundary flux profile (at $y=0$ ) for a bipolar arcade chosen as $\psi(x, y=0, t=0)=8 /\left((x / a)^{2}+3\right)$. The poloidal mag- 
netic flux of the hydrostatic equilibrium with the boundary condition of $\psi=0$ at infinity is given by $\psi(x, y, t=0)=$ $(8 / \sqrt{3})((y / a)+\sqrt{3}) /\left((x / a)^{2}+(y / a+\sqrt{3})^{2}\right)$. Note that $\psi$ is maximum at $x=0$ and decreases as $|x|$ increases. The maximum of $\left|B_{x}\right|$ is located at $x=0$ and $B_{x}$ changes sign at $x= \pm \sqrt{3} a$. In the following we consider $a=1$.

The photosphere modeled by the bottom boundary is considered as a perfect conductor such that the magnetic field is frozen into the plasma and magnetic field above the model photosphere cannot diffuse into it. Assuming no flows across the bottom boundary, the flux of the boundary normal magnetic field is conserved. This also implies the conservation of the poloidal flux in the simulation domain because the $\psi$ maximum, $\psi_{0}$, is located at the origin where the flow velocity is zero. At the bottom boundary, the shearing velocity $v_{z}$ is given as a function of the $x$-coordinate and time, $v_{z}(x, y=0, t)=f_{z}(t) V_{z}(x)$, where $V_{z}(x)=$ $V_{z 0} x \exp \left(\left(1-x^{2}\right) / 2\right)$. To avoid fast shock wave generation we choose the time dependent part $f_{z}(t)$ to change gradually between zero and one so that the duration of the shearing motion and its acceleration and deceleration periods are given by $f_{z}(t)=\left(t-\tau_{0}\right) /\left(\tau_{1}-\tau_{0}\right)$ for $\tau_{0} \leq t<\tau_{1}$, $f_{z}(t)=1$ for $\tau_{1} \leq t<\tau_{2}, f_{z}(t)=\left(t-\tau_{2}\right) /\left(\tau_{f}-\tau_{2}\right)$ for $\tau_{2} \leq t<\tau_{f}, f_{z}(t)=0$ otherwise. With the shearing velocity profile, the boundary normal field maxima are subject to the highest shearing velocity. The fluid elements at those locations thus travel in the $z$-direction the greatest distance from the initial locations and this distance, denoted by $\zeta_{m}(t)$, is $\zeta_{m}(t)=V_{z 0} \int_{0}^{t} f_{z}\left(t^{\prime}\right) d t^{\prime}$. The effect of this field line footpoint shearing motion is to create a toroidal magnetic field and increase the magnetic shear in the arcade field. Above a critical magnetic shear in the arcade field the field lines are compressed by the $\mathbf{J} \times \mathbf{B}$ from both side of the $x=0$ plane toward each other to form a current sheet, which is a necessary condition for magnetic reconnection to occur.

\section{Flaring Processes}

We have performed simulations to study the effects of general footpoint motions parallel and perpendicular to the polarity inversion line (Choe and Cheng, 2000). To concentrate on the flare mechanism, we only present simulation results with shearing footpoint motions parallel to the polarity inversion line. Simulation runs with different values of finite resistivity and shearing velocity show similar evolutionary features. A typical evolutionary trend is shown in Fig. 1 which shows constant poloidal flux lines at different times with $\eta=10^{-5}$ and $V_{z 0}=10^{-3} v_{0}$. As the magnetic shear is increased by the shearing footpoint motion, the current layer in the center of the magnetic arcade becomes thinner in the $x$-direction and longer in the $y$-direction so that magnetic reconnection takes place and a magnetic island is created. This result was reported by previous numerical simulation studies (Mikić and Linker, 1994; Choe and Lee, 1996; Amari et al., 1996). The magnetic reconnection is found to be triggered around $t \approx 11000 t_{0}$ which corresponds to $\zeta_{m} \approx 11$. The existence of a magnetic island in a low $\beta$ plasma depends crucially on the presence of toroidal magnetic field ( $B_{z}$ in our notation) in it. The toroidal magnetic field in the arcade under the magnetic island is reduced because the re- connected field lines in the underlying arcade have a smaller conjugate footpoint distance in the $z$-direction than the old field lines before magnetic reconnection occurs. Note that, although the conjugate footpoint distance may not be defined and diverge in the separatrix connected to the X-line (Choe and Lee, 1996), the toroidal flux in a finite flux volume does not diverge and the magnetic shear in the underlying arcade is always decreased after reconnection. Thus, the magnetic reconnection redistributes the toroidal flux previously contained in line-tied flux tubes into two new toroidal flux systems: one with the magnetic island and the other with the line-tied reconnected field. The differential toroidal flux and thus the magnetic shear in a reconnected field line are reduced after the reconnection occurs. By continuing the shearing motion, the magnetic shear in the underlying arcade is again increased and a new reconnection is initiated at $t \approx 19000 t_{0}\left(\zeta_{m} \approx 19\right)$ to create a new island. The newborn magnetic island rises and pushes up the line-tied field lines surrounding it so that these line-tied field lines start to reconnect with the field lines in the upper island through the upper X-line. After all the line-tied field lines in the underlying arcade surrounding the newborn island have reconnected with the upper island field lines, the two magnetic islands quickly coalesce to form one island. This merged island keeps on rising and gains flux as field lines surrounding it continue to reconnect in the vertical current sheet below.

A flaring event is considered to consist of the processes of magnetic reconnection to generate a new island, the newborn island's merging with an upper island, and the reconnection under the integrated island. Figure 1 shows that the generation of a new island and the subsequent merging of islands are repeated with some time interval while the shearing footpoint motion continues. This repetitive occurrence of a sequence of reconnection processes is interpreted as a set of homologous flares. New magnetic islands are created at $t \approx 11000 t_{0}\left(\zeta_{m} \approx 11\right), 19000 t_{0}\left(\zeta_{m} \approx 19\right), 29000 t_{0}$ $\left(\zeta_{m} \approx 29\right)$ and $40000 t_{0}\left(\zeta_{m} \approx 40\right)$, respectively. The island merging occurs in a relatively short time after a new island is born. The time interval between successive creation of new islands is thus around $10000 t_{0}$, and with $t_{0}=9.3 \mathrm{~s}$ it is about a day, although it has a mild tendency of increase with progress in island generation.

To investigate the kinematics of the island systems, the height of O-lines in the islands versus time is shown in Fig. 2. Compared with the first island and other integrated islands, newborn islands rise far faster. For example, the rising speed of the integrated island formed at $t \approx 20000 t_{0}$ is about $1.3 \times 10^{-3} v_{0}$ (with $v_{0}=3.2 \times 10^{3} \mathrm{~km} / \mathrm{s}$, this speed is about $4 \mathrm{~km} / \mathrm{s}$ ). On the other hand, the new island born at $t \approx 29000 t_{0}$ rises in the beginning at a speed of $\sim 3 \times 10^{-3} v_{0}$ (about $10 \mathrm{~km} / \mathrm{s}$ ) and in the merging stage at a speed of $\sim 2 \times 10^{-2} v_{0}$ (about $65 \mathrm{~km} / \mathrm{s}$ ). With the repetition of island generation and merging, the rising speeds of the integrated island and the newborn island increase. From other simulation runs with different resistivity, we have found that the rising speed of islands also depends on the reconnection rate. With resistivity of $5 \times 10^{-5}$, the rising speeds of newborn islands are more than twice of those for $\eta=10^{-5}$; with $\eta=10^{-3}$ they are almost ten times of those for $\eta=10^{-5}$. The rising speed of a newborn island in a merging process is 

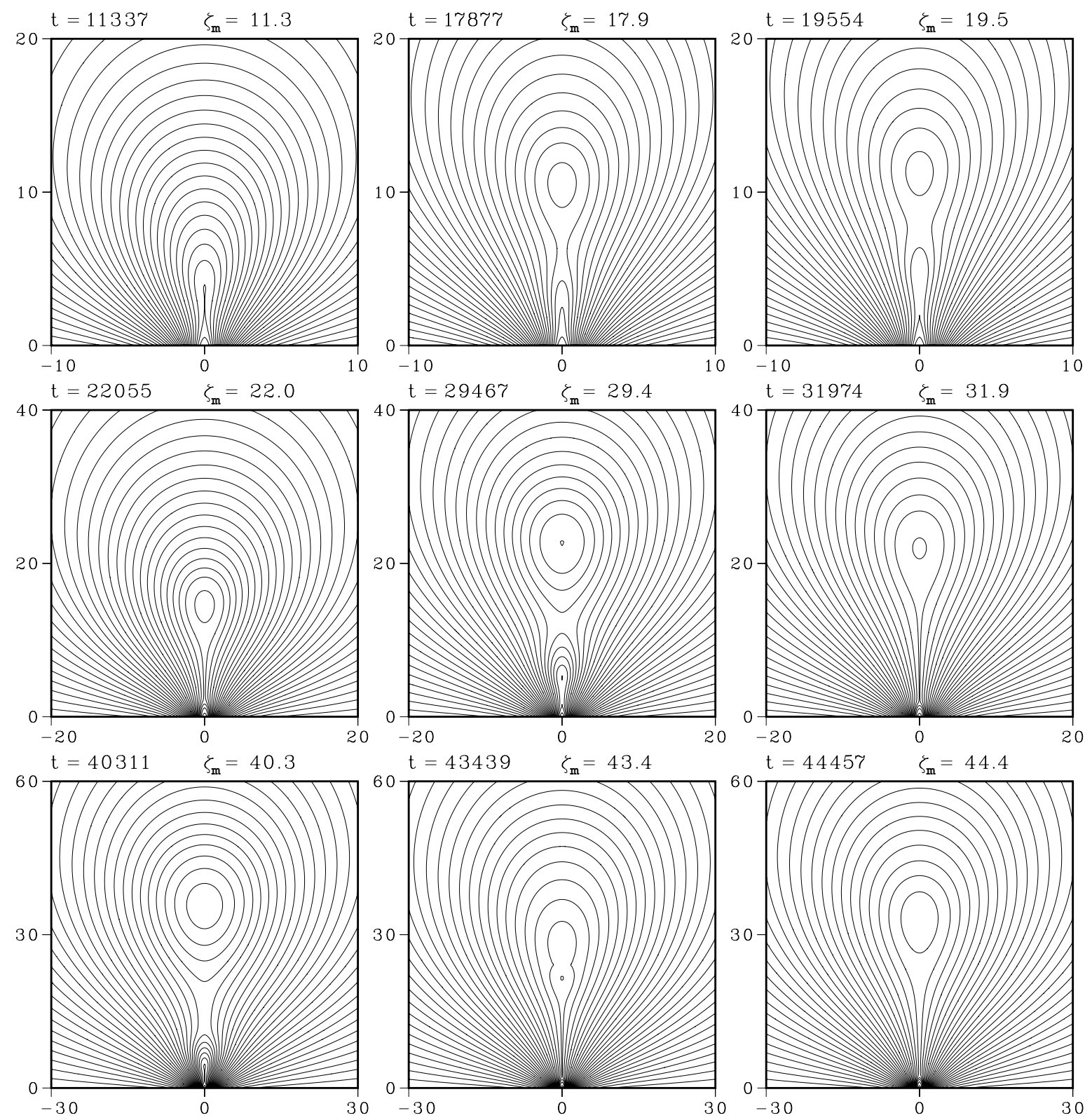

Fig. 1. Repetitive formation of magnetic islands and their merging under continuous footpoint shearing for $\eta=10^{-5}$. Field lines projected on the $x y$-plane are shown for different times. Note that the figure spatial scale increases row by row. The plasma displacement in the $z$-direction at the boundary normal field maxima $(x= \pm 1)$ is denoted by $\zeta_{m}$.

roughly proportional to $\eta^{1 / 2}$. However, it should be kept in mind that this result is obtained with a spatially uniform resistivity. As shown in previous MHD simulations (Choe and Lee, 1996), the reconnection rate can vary depending on the size of the diffusion region and the spatial profile of resistivity. Therefore, one should not give too much meaning to the above numbers, but should pay attention to the relative magnitudes.

The rising of a magnetic island is a consequence of magnetic reconnection. However, one should not confuse the rising speed of the island with the reconnection outflow speed, which is the Alfvén speed upstream of the current sheet. Generally, the island moves much slower than the reconnection outflow. This is because the line-tied arcade field surrounding the island hinders the island from moving freely. The force causing the magnetic island movement is deter- mined by the magnetic field enveloping the island (Choe and Lee, 1996) and the island motion is a part of the global process approaching a new equilibrium. The dynamics of magnetic islands can be qualitatively understood by considering the currents in the system. The attraction of two islands before and during coalescence is quite natural because they have toroidal currents $\left(J_{z}\right)$ of the same direction. The rising of a single island, whether a newborn or an integrated one, can be understood with the concept of an imaginary current lying below the solar surface (Van Tend and Kuperus, 1978). The magnetic field generated by coronal currents cannot permeate the photosphere because of the high conductivity and large inertia in the solar interior. The fixed flux boundary condition in our simulation is actually the implementation of this high inductance condition in the photosphere. In this situation, the Lorentz force acting on a current carry- 


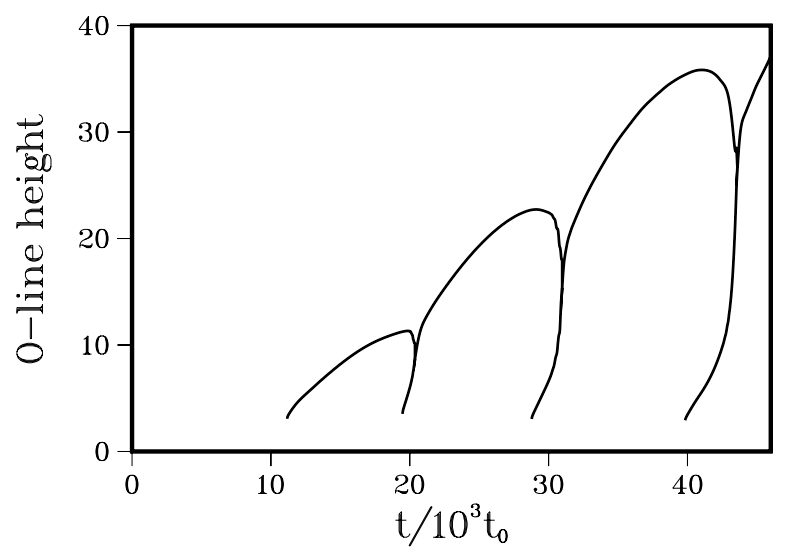

Fig. 2. The height of $\mathrm{O}$-lines of the magnetic islands in Case $1 \mathrm{~A}$ as a function of time. The slope of the curves represents the rising speed of the magnetic islands.

ing plasma can also be described as the force exerting on the coronal current by the image current of the opposite direction and the source current of the same direction generating the ambient potential field, both of which are located below the solar surface (Van Tend and Kuperus, 1978; Priest and Forbes, 1990; Forbes, 1990).

In Fig. 2, we should note that the motion of newborn islands consists of two phases: the newborn island rises rather slowly, although faster than the pre-existing one, in the first phase and then much faster in the second phase. The first phase mainly involves the early phase of reconnection during field compression and current sheet thinning. Dynamics in the second phase is mostly governed by magnetic reconnection between the upper island and the underlying flux system containing a newborn island. The rising speed of the newborn island thus depends on the reconnection rate. As can be noticed in the motion of the first island created at $t \approx 11000 t_{0}\left(\zeta_{m} \approx 11\right)$, a single island does not have the second phase with a faster motion. Although the altitude of the integrated island becomes higher with time, it has moved only a few solar radii in a few days in our simulation. This is because the 2D Cartesian geometry adopted in our simulation energetically inhibits the island system from totally escaping from the sun. In a more realistic 3D geometry, however, we expect the island to be accelerated more easily and be expelled farther away from the solar surface. We also note that the numerical study of magnetic reconnection in a linear force free field by (Magara et al., 1997) also showed an increase of the plasmoid speed before the maximum reconnection electric field is achieved. However, the change in the plasmoid velocity between the two phases is only about a factor of 2, which is much smaller than in our simulation (about a factor of 6.5 for $\eta=10^{-5}$ ).

We emphasize that in our simulations, two different types of reconnection processes are involved. The reconnection of line-tied arcade field lines takes place in a vertically elongated current sheet, creating a magnetic island and transferring magnetic fluxes to the island. The reconnection between the upper magnetic island and the underlying flux system containing a newborn island takes place in a horizontally elongated current sheet. This process comprises

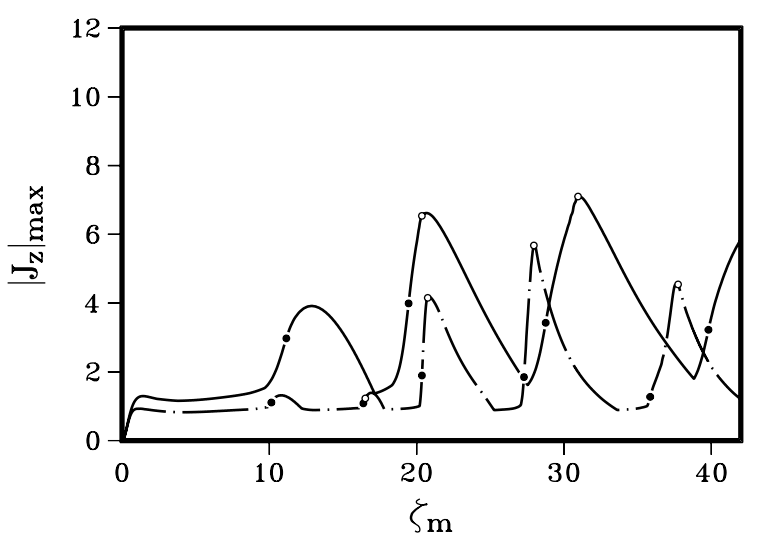

Fig. 3. The evolution of the maximum magnitude of the toroidal current $J_{z}$ in the current sheet under the magnetic island as a function of $\zeta_{m}$, which is the plasma displacement at $x= \pm 1$. The solid line represents the case with $V_{z 0}=10^{-3} v_{0}$ and $\eta=10^{-5}$, the chain-dotted line represents the case with $V_{z 0}=10^{-3} v_{0}$ and $\eta=5 \times 10^{-5}$.

the reconnection of the upper island with the line-tied field and the reconnection between two islands. The sequence of these reconnection processes is regarded to constitute an individual flaring event.

Now we investigate the relationship between these reconnection processes and their corresponding meanings in solar flares. From the evolution of magnetic energy we find that the magnetic energy released by merging of two islands is smaller than the energy released by the subsequent reconnection of line-tied field under the integrated island although the former process proceeds much faster than the latter. Thus, the main phase of a flare can be attributed to the reconnection in a vertically elongated current sheet under the integrated island as in conventional pictures of solar flares (e.g., Sturrock, 1968; Kopp and Pneuman, 1976; Tsuneta, 1996). Figure 3 shows the evolution of the maximum current density in the vertically elongated current sheet, $\left|J_{z}\right|_{\max }$. Because the reconnection electric field, given by $E_{z}=\eta J_{z}$ in the current sheet, equals to the poloidal flux reconnected per unit time, the $\left|J_{z}\right|_{\max }$ curve indicates how much flux is being reconnected in the X-line which is located near the current density maximum. As shown in Fig. 3, the solid line shows the maximum current density which increases before the initiation of reconnection in the underlying arcade due to the current sheet thinning, continues to increase during the reconnection processes, peaks at the time of island merging completion, and then slowly decays until a new current sheet is formed in the underlying arcade. The time interval from the reconnection trigger in the underlying arcade to the end of the slow island rising phase can be interpreted as the preflare phase. The flux reconnecting rate further increases in the fast island rising phase and reaches a maximum when merging of the two islands is completed. This rather short time interval is identified with the impulsive (or flash) phase. The highest flux reconnecting rate in this phase is attributed to the rapid upward motion of the lower island that elongates the line-tied field wrapping around both islands to form a very thin current sheet. After the merging of two islands completes, reconnection of line-tied field continues, but slows down with decreasing $\left|J_{z}\right|_{\max }$. This phase 


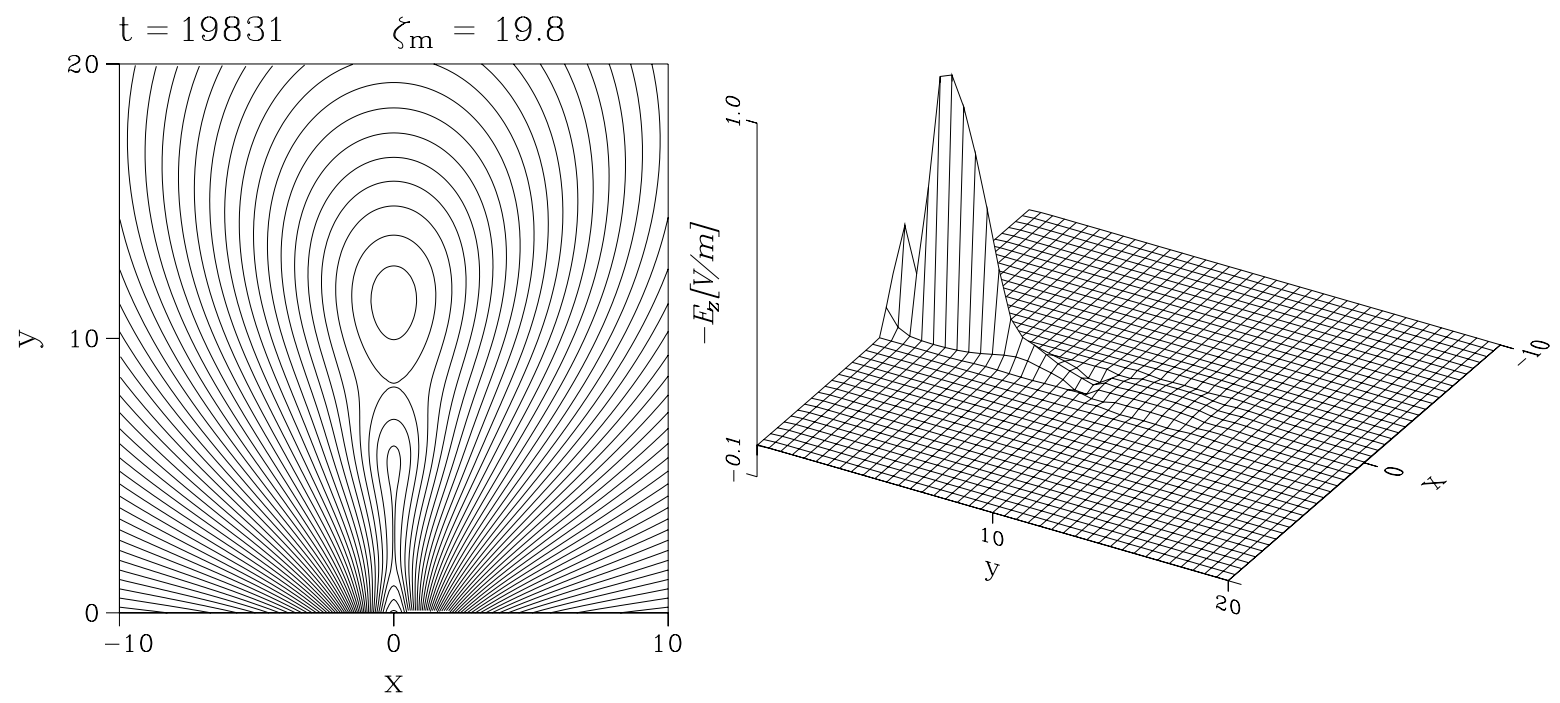

Fig. 4. The poloidal magnetic flux contours and the reconnection electric field $E_{z}$ distribution in the $(x, y)$ plane at $t=19831 t_{0}\left(\zeta_{m} \approx 19.8\right)$.

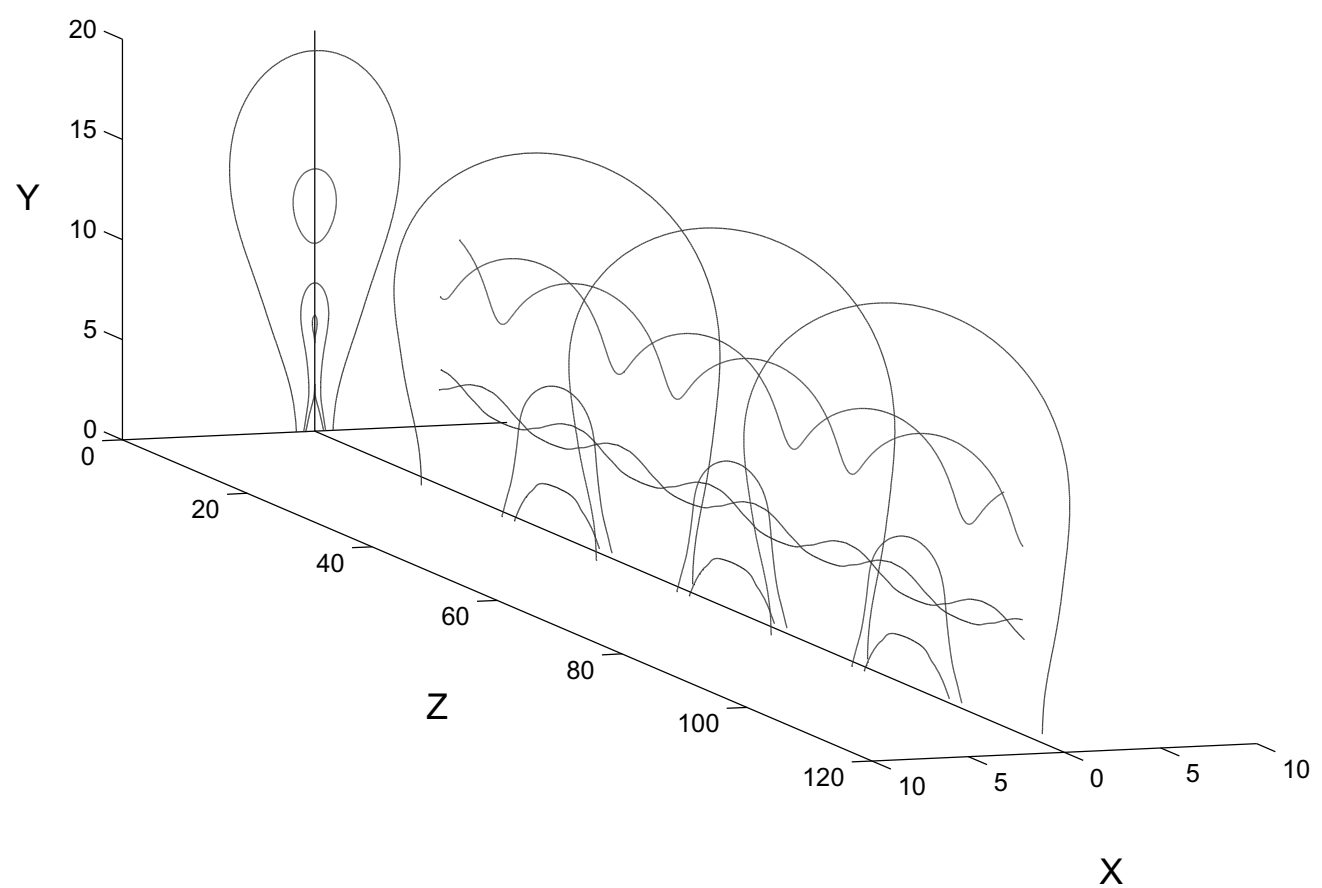

Fig. 5. The 3D magnetic field lines and their projection onto the $(x, y)$ plane at $t=19831 t_{0}\left(\zeta_{m} \approx 19.8\right)$.

is longer than the former two phases and is considered as the main phase of a flare. The dependence of the time span of a flaring event on reconnection rate can also be seen from the chain-dotted line in Fig. 3, which represents the maximum current density for the case with a larger resistivity of $\eta=5 \times 10^{-5}$. The flaring event occurs in a much shorter time period than in the smaller resistivity case, but the time interval between successive flaring events is approximately unchanged.

To know how electrons are accelerated to high velocity we need to know the 3D magnetic field line structure as well as the reconnection electric field distribution in the $(x, y)$ plane. From Fig. 3, we can obtain the maximum reconnec- tion electric field $E_{z, \max }$. For $\eta=10^{-5}, E_{z, \max } \approx 0.64 \mathrm{~V} / \mathrm{m}$ at the time of the second island formation $\left(t \approx 1.9 \times 10^{4} t_{0}\right)$ when $\left|J_{z}\right|_{\max } \approx 4.0 J_{0}$ and $E_{z, \max } \approx 1.1 \mathrm{~V} / \mathrm{m}$ at the completion of island merging when $\left|J_{z}\right|_{\max } \approx 6.7 J_{0}$. Figure 4 shows the poloidal magnetic flux distribution and the corresponding $\left|E_{z}\right|$ distribution in the $(x, y)$ plane at $t=19831 t_{0}$ $\left(\zeta_{m} \approx 19.8\right)$. We note that $\left|E_{z}(x, y)\right|$ is localized near the reconnection site in the current sheet under the newborn island. $\left|E_{z}\right|$ is maximum at $y=2.5, x=0$, decreases toward larger $y$ and the photosphere, and decreases rapidly as $|x|$ increases. Figure 5 shows the 3D magnetic field lines and their projection onto the $(x, y)$ plane. The field lines in the plasmoids clearly show helical structure. The field lines 
overlying the plasmoids show arcade structure. But the field lines that form current sheet below the reconnection $X$-point show a dominant toroidal $(z)$ component in the current sheet; these field lines are almost along the $z$-direction in the current sheet region for a long distance and connect to the photosphere with a shorter distance. With an electric field of 1.1 $\mathrm{V} / \mathrm{m}$, electrons can be accelerated along the flat top portion of the field lines to an energy of $11 \mathrm{keV}$ in $10 \mathrm{~km}$. However, we must bear in mind that this reconnection electric field is obtained with an anomalous resistivity $\left(\eta=10^{-5}\right)$, which is much larger than the classical resistivity in the actual solar corona ( $\eta \sim 10^{-12}$ or smaller). Theories of magnetic reconnection are still being developed to determine the anomalous resistivity and the resistivity scaling of the electric field is still unknown. However, if we assume a tearing mode type scaling with the electric field proportional to $\eta^{3 / 5}$, the peak reconnection electric field would be roughly $1.1 \times 10^{-3} \mathrm{~V} / \mathrm{m}$ for an anomalous resistivity of $\eta=10^{-10}$ at the completion of island merging. With this electric field, electrons can be accelerated to an energy of $10 \mathrm{keV}$ in $10,000 \mathrm{~km}$, a distance which is still smaller than a typical flaring arcade size. Thus, high energy electrons responsible for X-ray emission can be generated by the reconnection electric field with our flare model.

\section{Summary and Discussion}

In this paper, we have presented a solar flare model based on the evolution of magnetic arcades under various shearincreasing footpoint motions, focusing on dynamical interaction between magnetic islands and between a magnetic island and an ambient arcade. The arcade evolution under more general footpoint shearing, diverging and converging motions (and combination of these motions) in the photosphere can be found in the paper by Choe and Cheng (2000). In particular, this study demonstrates repetitive flaring events in solar active regions, i.e., homologous flares. Many physical processes revealed in our study are believed to be involved in general solar flares. We found that when a magnetic arcade is subject to a continuing shear-increasing footpoint motion, generation of magnetic islands via magnetic reconnection and their coalescence can repeatedly take place with some time interval. The series of these reconnection processes is regarded to constitute a sequence of homologous flares.

The rising motion of a newborn island comprises a slower first phase and a faster second phase. The first phase involves arcade field reconnection creating and adding flux to the new island and is identified with the preflare phase. Coalescence of two magnetic islands takes place within a short time span in the second phase, which is identified with the impulsive (or flash) phase of a flare. The reconnection electric field in the current sheet under a newborn island increases with the rising of the island. The reconnection electric field obtained from our simulations is large enough to accelerate electrons to an energy much larger than $10 \mathrm{keV}$ for X-ray emission. The merging of two islands creates a long and thin current sheet below and facilitates reconnection of the line-tied field. This phase of arcade field reconnection under the integrated island persists much longer than the impulsive phase. Although the reconnection elec- tric field decays during this phase, more magnetic energy is released in total. This phase is identified with the main phase of a flare.

It should be mentioned that the role of the upper magnetic island in our flare model can be performed by any magnetic flux system lying above the underlying arcade. The upper flux system may be connected to the interplanetary magnetic field or to distant magnetic poles on the solar surface. Thus, our flare model can explain the Yohkoh observation by Ohyama and Shibata (1997) that the plasmoid motion comprises a slower first phase and a faster second phase. Such two phases of rising velocity could not be interpreted tailored to the conventional picture of plasmoid formation as in previous studies of Mikić and Linker (1994), Choe and Lee (1996), and Amari et al. (1996). Our numerical result indicates that the faster second phase can be naturally achieved if the closed field lines above the plasmoid are reconnected with the further overlying flux. Most flare energy in our simulation is released by the reconnection of line-tied field in a vertically elongated current sheet although the island coalescence is a more rapid process. Thus, our simulation results do not support the flux tube merging model of flares by Gold and Hoyle (1960). However, the rapid rising of the magnetic island in a merging process plays a significant role in the fast reconnection in the impulsive (or flash) phase by stretching the arcade field lines so much as to form a thin and long current sheet. This is consistent with the plasmoid-induced reconnection model of solar flares proposed by Shibata (1998). Our model, however, provides a plasmoid acceleration mechanism which was not given in Shibata's model.

Acknowledgments. This work is supported by the DoE Contract No. DE-AC02-76-CHO3073 and the NSF grant ATM-9906142.

\section{References}

Amari, T., J. F. Luciani, J. J. Aly, and M. Tagger, Plasmoid formation in a single sheared arcade and application to coronal mass ejections, Astron. Astrophys., 306, 913-923, 1996.

Anzer, U., Models of structure and dynamics of prominences, in Physics of Solar Prominences, IAU Colloquium 44, edited by E. Jensen, P. Maltby, and F. Q. Orrall, p. 322, Blindern-Oslo, Institute of Theoretical Astrophysics, 1979.

Cheng, C. Z. and G. S. Choe, Current sheets and prominence formation in the solar atmosphere, Astrophys. J., 505, 376-389, 1998.

Choe, G. S. and C. Z. Cheng, A model of solar flares and their homologous behavior, Astrophys. J., 541, 449-467, 2000.

Choe, G. S. and L. C. Lee, Formation of solar prominences by photospheric shearing motions, Solar. Phys., 138, 291-329, 1992.

Choe, G. S. and L. C. Lee, Evolution of solar magnetic arcades. II. Effect of resistivity and solar eruptive processes, Astrophys. J., 472, 372-388, 1996

Forbes, T. G., Numerical simulation of a catastrophe model for coronal mass ejections, J. Geophys. Res., 95, 11919-11931, 1990.

Gold, T. and F. Hoyle, On the origin of solar flares, Mon. Not. R. Aston. Soc., 120, 89-105, 1960.

Inhester, B., J. Birn, and M. Hesse, The evolution of line-tied coronal arcades including a converging footpoint motion, Solar. Phys., 138, 257 281, 1992.

Kane, S. R., Impulsive flash phase of solar flares: Hard X-ray, microwave, EUV and optical observations, in Coronal Disturbances, IAU Symposium No. 57, edited by G. A. Newkirk, p. 105, Dordrecht, D. Reidel, 1974.

Kopp, R. A. and G. W. Pneuman, Magnetic reconnection in the corona and the loop prominence phenomenon, Solar. Phys., 50, 85-98, 1976.

Kuperus, M. and M. A. Raadu, The support of prominences formed in neutral sheets, Astron. Astrophys., 31, 189-193, 1974. 
Linker, J. A. and Z. Mikić, Disruption of a helmet streamer by photospheric shear, Astrophys. J., 438, L45-48, 1995.

Magara, T., K. Shibata, and T. Yokoyama, Evolution of eruptive flares. I. Plasmoid dynamics in eruptive flares, Astrophys. J., 487, 437-446, 1997.

Masuda, S., T. Kosugi, H. Hara, S. Tsuneta, and Y. Ogawara, A loop-top hard X-ray source in a compact solar flare as evidence for magnetic reconnection, Nature, 371, 495-497, 1994.

Mikić, Z. and J. A. Linker, Disruption of coronal magnetic arcades, Astrophys. J., 430, 898-912, 1994.

Mikić, Z., D. C. Barnes, and D. D. Schnack, Dynamical evolution of a solar coronal magnetic field arcade, Astrophys. J., 328, 830-847, 1988.

Ohyama, M. and K. Shibata, Preflare heating and mass motion in a solar flare associated with hot plasma ejection: 1993, November 11 C9.7 flare, Pub. Astron. Soc. Japan, 49, 249-261, 1997.

Priest, E. R., Solar Magnetohydrodynamics, D. Reidel, Dordrecht, 1982.

Priest, E. R. and T. G. Forbes, Magnetic field evolution during prominence eruptions and two-ribbon flares, Solar. Phys., 126, 319-350, 1990.

Shibata, K., A unified model of solar flares, in Observational Plasma As- trophysics: Five Years of Yohkoh and Beyond, edited by T. Watanabe, T. Kosugi, and A. C. Sterling, p. 187, Boston, Kluwer Academic Publishers, 1998.

Shibata, K., S. Masuda, M. Shimojo, H. Hara, T. Yokoyama, S. Tsuneta, T. Kosugi, and Y. Ogawara, Hot-plasma ejections associated with compact-loop solar flares, Astrophys. J., 451, L83-85, 1995.

Sturrock, P. A., A model of solar flares, in Structure and Development of Solar Active Regions, IAU Symposium No. 35, edited by K. O. Kiepenheuer, p. 471, Dordrecht, D. Reidel, 1968.

Tsuneta, S., Structure and dynamics of magnetic reconnection in a solar flare, Astrophys. J., 456, 840-849, 1996.

Van Tend, W. and M. Kuperus, The development of coronal electric current systems in active regions and their relation to filaments and flares, Solar. Phys., 59, 115-127, 1978.

C. Z. Cheng (e-mail: fcheng@pppl.gov) and G. S. Choe 